\title{
Pancreatic duodenal homeobox 1 protein is a novel $\beta$-cell-specific autoantigen for type I diabetes
}

\author{
Shi-Wu Li ${ }^{1}$, Vijay Koya ${ }^{1}$, Yi Li ${ }^{2}$, William Donelan${ }^{1}$, Peng Lin ${ }^{1}$, Westley H Reeves ${ }^{2}$ and Li-Jun Yang ${ }^{1}$
}

Pancreatic duodenal homeobox $1(\mathrm{Pdx} 1)$ protein is a key transcription factor involved in the regulation of insulin gene expression that is expressed at high levels in the $\beta$-cells of the pancreatic islets. We asked whether Pdx 1 is a target of antiislet autoimmunity in type I diabetes (T1D). Pdx1 autoantibodies (PAAs) were detected in non-obese diabetic (NOD) mice using ELISA, western blotting, and radioimmunoprecipitation of $\left[{ }^{35} \mathrm{~S}\right]$-labeled insulinoma cell line-derived Pdx 1 protein. PAAs were detected as early as at 5 weeks of age, and generally peaked before the onset of clinically overt diabetes in diabetes-prone female NOD mice. Levels declined substantially after the onset of diabetes. PAAs were not detected in the sera of NOD-scid, C57BL/6, or BALB/C mice. The titers of PAAs in NOD mouse sera were as high as 1/93750 by ELISA. The fine specificity of PAAs was determined by western blotting using a series of truncated recombinant Pdx 1 proteins. The immunodominant epitopes were located to the C-terminus of the Pdx1 (p200-283) in NOD mice. PAAs also were detected in sera from human T1D patients, but the major epitopes were localized to amino acids 159-200 as well as the same region (p200-283) recognized by PAAs from NOD mice. Using $\left[{ }^{3} \mathrm{H}\right]$ thymidine incorporation, the p83 fragment of Pdx1 specifically stimulated proliferation of splenic T cells from recent-onset diabetic NOD mice. The presence of PAAs in prediabetic NOD mice and human T1D patients, and Pdx1-specific T-cell proliferation in NOD mice provide a strong rationale for further investigation of the pathogenic role of immune responses against Pdx1 in T1D.

Laboratory Investigation (2010) 90, 31-39; doi:10.1038/labinvest.2009.116; published online 9 November 2009

KEYWORDS: Pdx1; autoantigen; autoimmunity; type I diabetes

Type 1 diabetes (T1D) is an autoimmune disease characterized by the development of autoantibodies and destructive T-cell infiltration of insulin-producing islet $\beta$-cells. ${ }^{1}$ Several candidate autoantigens have been identified and autoantibody production may precede disease in family members of T1D patients and other individuals at risk for developing $\mathrm{T}_{1 D}{ }^{2-5}$ The non-obese diabetic (NOD) mouse is an important animal model of autoimmune diabetes. ${ }^{6,7}$ Disease occurs spontaneously and shares many phenotypic and genetic similarities with T1D in humans. ${ }^{6,7}$ Lymphocyte infiltration of the islets of Langerhans begins at 4 weeks of age, progressing from peri-insulitis to severe insulitis by 10 weeks of age. ${ }^{6,7}$ The onset of diabetes typically ensues at $12-14$ weeks in female NOD mice. To date, there is no effective immunotherapy to treat or prevent human T1D. ${ }^{5,8-10}$

We have previously shown that treatment of streptozotocin (Stz)-induced murine diabetes with recombinant pancreatic duodenal homeobox $1(\mathrm{Pdx} 1)$ protein $(\mathrm{rPdx} 1)$ reverses diabetes by stimulating $\beta$-cell regeneration and liver cell reprogramming into insulin-producing cells. ${ }^{11}$ It has been reported that in NOD mice treated with Pdx1 delivered by an adenovirus vector, the autoimmune attack on the pancreatic islets can be downregulated. ${ }^{12}$ Recently, we found that treatment of prediabetic female NOD mice with $\mathrm{rPdx} 1$ or with a non-functional mutant $\mathrm{Pdx} 1$ protein prevented the onset of diabetes (unpublished observation). In view of the modulation of diabetes by this mutant protein, in this study we tested the hypothesis that Pdx1 might be an autoantigen relevant to pathogenesis of T1D in NOD mice. Our data indicate that $\mathrm{Pdx} 1$ is a novel $\beta$-cell-specific autoantigen that stimulates an antigen-specific T-cell response and is recognized by autoantibodies present in serum samples from both prediabetic and diabetic NOD mice and a subset of T1D patients. High levels of Pdx1 autoantibodies (PAAs) were

\footnotetext{
${ }^{1}$ Department of Pathology, Immunology, and Laboratory Medicine, University of Florida College of Medicine, Gainesville, FL, USA and ${ }^{2}$ Division of Rheumatology \& Clinical Immunology, Department of Medicine, University of Florida College of Medicine, Gainesville, FL, USA

Correspondence: Dr L-J Yang, MD, PhD, Department of Pathology, Immunology, and Laboratory Medicine, University of Florida College of Medicine, Box 100275 Gainesville, FL 32610-0275, USA.

E-mail: yanglj@pathology.ufl.edu

Received 4 June 2009; revised 27 July 2009; accepted 12 August 2009
} 
detected in young NOD mice and the peak of PAA often occurred before the onset of diabetes.

\section{MATERIALS AND METHODS} Serum Samples and Animals

Serum samples were collected from female NOD mice and congenic nondiabetic NOD-scid, BALB/c, and C57BL/6 mice (Jackson Laboratory, Bar Harbor, ME, USA). Mice were housed in an SPF facility at the University of Florida. Serum samples from female NOD mice were tested for PAAs at ages ranging from 5 to 25 weeks. Blood glucose was determined weekly starting at 10 weeks of age. Collection of serum samples from human subjects was approved by the Institutional Review Boards, and all animal experiments were approved by the Institutional Animal Care and Use Committee of the University of Florida.

\section{Preparation of Recombinant Pdx1, Mutant Pdx1, and Truncated Proteins}

Complementary DNA fragments of rat or human Pdx1 were inserted into the expression vector pET28 (Invitrogen, Carlsbad, CA, USA) and 6X histidine-tagged recombinant Pdxl, mutant Pdx1 lacking the protein transduction domain $(\mathrm{Pdx} 1 \Delta \mathrm{PTD})$, and truncated Pdx1 proteins were produced, purified, and characterized as previously described. ${ }^{11}$ In brief, C-terminal Pdx1 truncations were generated by site-directed mutagenesis using a QuikChange Kit (Stratagene) by introducing a stop codon (TAG) mutation into rat Pdx1 cDNA at the amino-acid position 120,160 , or 200 . The mutant cDNAs were verified by DNA sequence analysis. Three truncated Pdx1 fragments (1-119, 1-159, and 1-199) were expressed in the pET28 expression vector in Escherichia coli BL21(DE3) cells and purified by Ni-NTA affinity chromatography as described. ${ }^{11}$

\section{Expression and Purification of GST-P83 and GST-P40}

Rat Pdx1, amino acids 200-283 (p83), and Pdx1, amino acids 160-199 (p40), were expressed in E. coli as fusion proteins to glutathione- $S$-transferase (GST). A fragment of the cDNA encoding the amino acids $200-283$ or 160-199 of Pdx1 was amplified by PCR and ligated into the BamHI/XhoI sites of pGEX-5x-1 expression vector (Promega) for generating fusion proteins GST-p83 and GST-p40, respectively. A 6X histidine tag was attached to the N-terminus of GST-p40 to facilitate purification of this insoluble protein. The plasmids were transformed into E. coli BL21 (DE3) cells and the fusion proteins were produced as described. ${ }^{11}$ GST and GST-p83 fusion proteins were purified using a glutathione-Sepharose affinity purification kit (Pierce, Rockford, IL, USA). Bound protein was eluted with $10 \mathrm{mM}$ reduced glutathione in $50 \mathrm{mM}$ Tris- $\mathrm{HCl}$ ( $\mathrm{pH} 8.0)$ and dialyzed against PBS. GSTp40 was purified by Ni-NTA affinity chromatography under denaturing conditions as described. ${ }^{11}$ Protein concentration was determined and the purified proteins were snap-frozen and stored in aliquots at $-80^{\circ} \mathrm{C}$ as described. ${ }^{11}$

\section{SDS-PAGE and Western Blot}

Pdx 1 and its truncated forms were separated by 12\% SDSpolyacrylamide gel electrophoresis (PAGE) and transferred onto nitrocellulose membranes (Bio-Rad). Blots were probed with mouse or human sera sample or with rabbit anti-Pdx1 antibodies (1:1000) as described, ${ }^{11,13}$ with slight modifications. The membrane was blocked with 5\% nonfat dry milk (Bio-Rad) in Tris-buffered saline (TBS, $\mathrm{pH}$ 7.5; Bio-Rad), and then incubated with sera from mice (1:1500 dilution) or human T1D patients (1:200 dilution) overnight at $4^{\circ} \mathrm{C}$. After washing five times with TBS containing $0.1 \%$ Tween-20, the membrane was incubated with horseradish peroxidase (HRP)-conjugated goat anti-mouse-IgG (1:4000, Abcam, Cambridge, MA, USA) or anti-human IgG (1:2000, South Biotech) for $30 \mathrm{~min}$ at $22^{\circ} \mathrm{C}$. Binding was detected by chemiluminescence (Amersham, Piscataway, NJ, USA).

\section{ELISA for Autoantibodies against Pdx1}

Autoantibodies against Pdx1 were quantified by ELISA. In brief, a microtiter plate (Nunc MaxiSorp, Fisher) was coated with $100 \mu \mathrm{l}$ of $\mathrm{rPdx} 1(10 \mu \mathrm{g} / \mathrm{ml})$ overnight at $4^{\circ} \mathrm{C}$. After washing the plate three times with PBS, the plate was incubated with $200 \mu \mathrm{l}$ of $5 \%$ dry milk in PBS (blocking buffer) for $1 \mathrm{~h}$. Next, mouse sera were added in duplicate at a 1:30 dilution or after serial dilutions in the blocking buffer for $1 \mathrm{~h}$. The bound antibodies were incubated with HRP-conjugated goat anti-mouse IgG antibodies (1:4000 in blocking buffer, Abcam) or HRP-goat-anti-mouse IgG isotype-specific antibody kit (IgG1-, IgG2a-, IgG2b-, and IgG3-specific; Santa Cruz Biotechnology, Santa Cruz, CA, USA) for $1 \mathrm{~h}$ at $22^{\circ} \mathrm{C}$. After washing five times, the plate was incubated with $100 \mu \mathrm{l}$ of substrate solution (BD OptEIA, BD Biosciences Pharmingen, San Diego, CA, USA) and developed at $22^{\circ} \mathrm{C}$ for $10 \mathrm{~min}$. The reaction was terminated by adding $50 \mu \mathrm{l}$ of $4 \mathrm{M}$ $\mathrm{H}_{2} \mathrm{SO}_{4}$, and absorbance was determined at $450 \mathrm{~nm}$. A positive result was defined as an $\mathrm{OD}_{450 \mathrm{~nm}} \geqslant 0.1$ (the mean of $\mathrm{BALB} / \mathrm{c}$ sera or istotype controls +3 s.d.). Intra- and interassay variation $(\mathrm{CV} \%)$ was determined with the same lot of ELISA plates and the same serum-positive and serum-negative samples.

\section{$\left[{ }^{35} \mathrm{~S}\right]$-Labeling and Immunoprecipitation}

Rat insulinoma INS-1 cells (clone 832/13, a gift of Christopher B Newgard, Duke University) were metabolically labeled overnight with $\left[{ }^{35} \mathrm{~S}\right]$ methionine plus $\left[{ }^{35} \mathrm{~S}\right]$ cysteine (GE Healthcare, Piscataway, NJ, USA) as described. ${ }^{14}\left[{ }^{35} \mathrm{~S}\right]$-labeled INS-1 cell lysate containing rat Pdx1 protein $(200 \mu \mathrm{l}$ volume, $10^{6}$ cell equivalents) were incubated with preformed complexes of immunoglobulin/protein A/G-Sepharose for $2 \mathrm{~h}$ at $4{ }^{\circ} \mathrm{C}$. Protein A/G-Sepharose was incubated with $10 \mu \mathrm{l}$ of mouse sera or Pdx1-treated mouse serum (prepared in our lab) overnight. After centrifuging, $20 \mu \mathrm{l}$ of $50 \%$ protein A/GSepharose beads (Pharmacia) were added followed by incubation at $22^{\circ} \mathrm{C}$ for $60 \mathrm{~min}$. Immune complexes were collected by centrifugation and washed three times. Proteins 
were freed by boiling in a sample buffer and analyzed on $12.5 \%$ SDS-polyacrylamide gels. The gels were stained and then fluorographed, followed by the exposure to X-ray film for 1 week as previously described. ${ }^{15}$ Similar immunoprecipitation (IP) was performed using unlabeled INS-1 cell lysates, and the Pdx1 protein from the cell lysates immunoprecipitated by the tested mouse sera was probed by western blotting using rabbit anti-Pdx1 polyclonal antibodies (1:2000 dilution). ${ }^{11}$

\section{T-Cell Proliferation Assay}

New-onset diabetic female NOD mice were killed and splenocytes were harvested. The splenocytes $\left(10^{6}\right.$ cells/well in triplicate) were incubated for $72 \mathrm{~h}$ at $37^{\circ} \mathrm{C}$ in a humidified atmosphere with $5 \% \mathrm{CO}_{2}$ in the absence or presence of various stimuli (see Results). Anti-CD3 antibody treatment was used as a positive control. T-cell proliferation was quantified by the incorporation of $\left[{ }^{3} \mathrm{H}\right]$ thymidine (Amersham, $1 \mu \mathrm{Ci} /$ well) for the last $24 \mathrm{~h}$ of incubation. The stimulation index (SI) was calculated as the ratio of the mean c.p.m. of antigen- or mitogen-treated cells divided by the mean c.p.m. of control cells cultured with medium alone. ${ }^{12}$

\section{Statistical Analysis}

Statistical analysis was carried out using the two-sample Student's $t$-test assuming unequal variances. A $P$-value $<0.05$ was considered significant.

\section{RESULTS}

\section{Discovery of Anti-PAAs in NOD Mouse Sera}

As part of a follow-up study for our recently published work showing that Pdx1 treatment reverses Stz-induced diabetes in $\mathrm{BALB} / \mathrm{c}$ mice, ${ }^{16}$ we investigated the effect of $\mathrm{Pdx} 1$ treatment in NOD mice. To examine whether daily Pdx1 treatment of NOD mice could stimulate the production of anti-Pdx1 antibodies, we first established and validated an ELISA for detecting anti-Pdx1 antibodies using highly purified rat or human $\mathrm{rPdx} 1$ as antigen and rabbit polyclonal antibodies to $\mathrm{Pdx}^{11}$ as a positive control. We then tested serum samples collected from pretreated, post-rPdx1-treated, and salinetreated (control) female NOD mice for the presence of antiPdx1 antibodies (Figure 1a). As expected, serum samples collected from 23-week-old mice treated with Pdx1 protein for 12 weeks showed strong immunoreactivity to Pdx1 antigen, consistent with a humoral immune response to rPdx1. Surprisingly, some serum samples from prediabetic (pretreatment) and control mice were also strongly positive. This result raised the possibility that Pdx1 might be an islet cell autoantigen in NOD mice, leading to the production of PAAs.

To test this hypothesis, we examined serum samples from several mouse strains including $\mathrm{C} 57 \mathrm{BL} / 6, \mathrm{BALB} / \mathrm{c}$, and nondiabetic congenic NOD-scid mice for the presence of PAAs. Figure $1 \mathrm{~b}$ shows that $54 \%$ of prediabetic female NOD mice had PAAs detectable at the screening dilution of $1: 30$, whereas no reactivity was seen in other mouse strains. These results indicated that PAAs were produced selectively by prediabetic NOD mice. Serial dilution of selected positive NOD mouse sera at age 10 weeks showed that the titers of these PAAs were as high as 1:93750 (Figure 1c). Induced anti-Pdx1 antibodies in immunized NOD mice and spontaneous antibodies in un-immunized PAA-positive $(\mathrm{PAA}+)$ NOD mice consisted of various isotypes (Figure 1d). IgG1 and IgG2b anti-Pdx1 antibodies predominated in the response to immunization as well as in the spontaneous response, whereas IgG3 PAAs were less abundant (Figure 1d). The levels of anti-Pdx1 IgG2b antibodies were considerably more variable in immunized mice than in the mice with spontaneous PAAs. As NOD mice express the $\operatorname{Igh} 1^{\mathrm{b}}$ allele, they express IgG2c instead of IgG2a. ${ }^{17}$ Thus, anti-Pdx1 IgG2 $\mathrm{c}^{1}$ antibody levels could not be accurately measured using the current commercial kit.

\section{PAAs also are Detectable by Western Blot and IP}

To further establish the existence of PAAs in NOD mice, selected PAA + or PAA-negative NOD sera, negative control $(\mathrm{BALB} / \mathrm{c})$ serum, and positive control rabbit polyclonal immune serum (rPdx1-IS) were used for western blotting using purified rat Pdx1 as antigen (Figure 2a). ELISA-PAA + NOD mouse sera detected a single band at $46 \mathrm{kDa}$ (lane 3), whereas ELISA-negative serum samples from BALB/c (lane 1) or NOD (lane 4) mice did not. In contrast, the immune serum (lane 2) showed a major band at $46 \mathrm{kDa}$ as well as several minor bands, possibly due to antibodies against minor proteins contaminating the Pdx1 antigen preparation used for immunization and/or degradation fragments of Pdx1. In addition, autoantibody specificity was verified by immunoprecipitating $\left[{ }^{35} \mathrm{~S}\right]$-labeled native Pdx1 from rat insulinoma INS-1 cell extracts. A band at $46 \mathrm{kDa}$ was immunoprecipitated by both NOD mouse Pdx1-treated serum (m-Pdx1-IS) (lane 2) and the ELISA-positive PAA serum (lane 3), whereas no clear band was detected in PAA-negative or control sera (Figure 2b, left panel). The identity of this immunoprecipitated protein band was confirmed by western blotting using rabbit anti-Pdx1 polyclonal antibodies to probe the membrane (Figure $2 \mathrm{~b}$, right panel). Thus, PAAs could be detected by western blotting and IP in addition to ELISA.

\section{PAAs Emerge before the Onset of Diabetes}

To determine whether the PAAs can predict disease onset, the relationship between PAAs and hyperglycemia was explored (Figure 3). Five-week-old female NOD mice $(n=20)$ were studied longitudinally and blood samples were taken biweekly until the onset of diabetes. Blood glucose levels were monitored weekly beginning at 10 weeks of age. PAAs were first detected at 5-15 weeks of age, and their levels gradually increased, peaked, and then declined over the next 8-12 weeks. PAAs often decreased to lower positive levels or disappeared completely after the onset of diabetes and the peak levels often preceded disease onset. In general, there was 

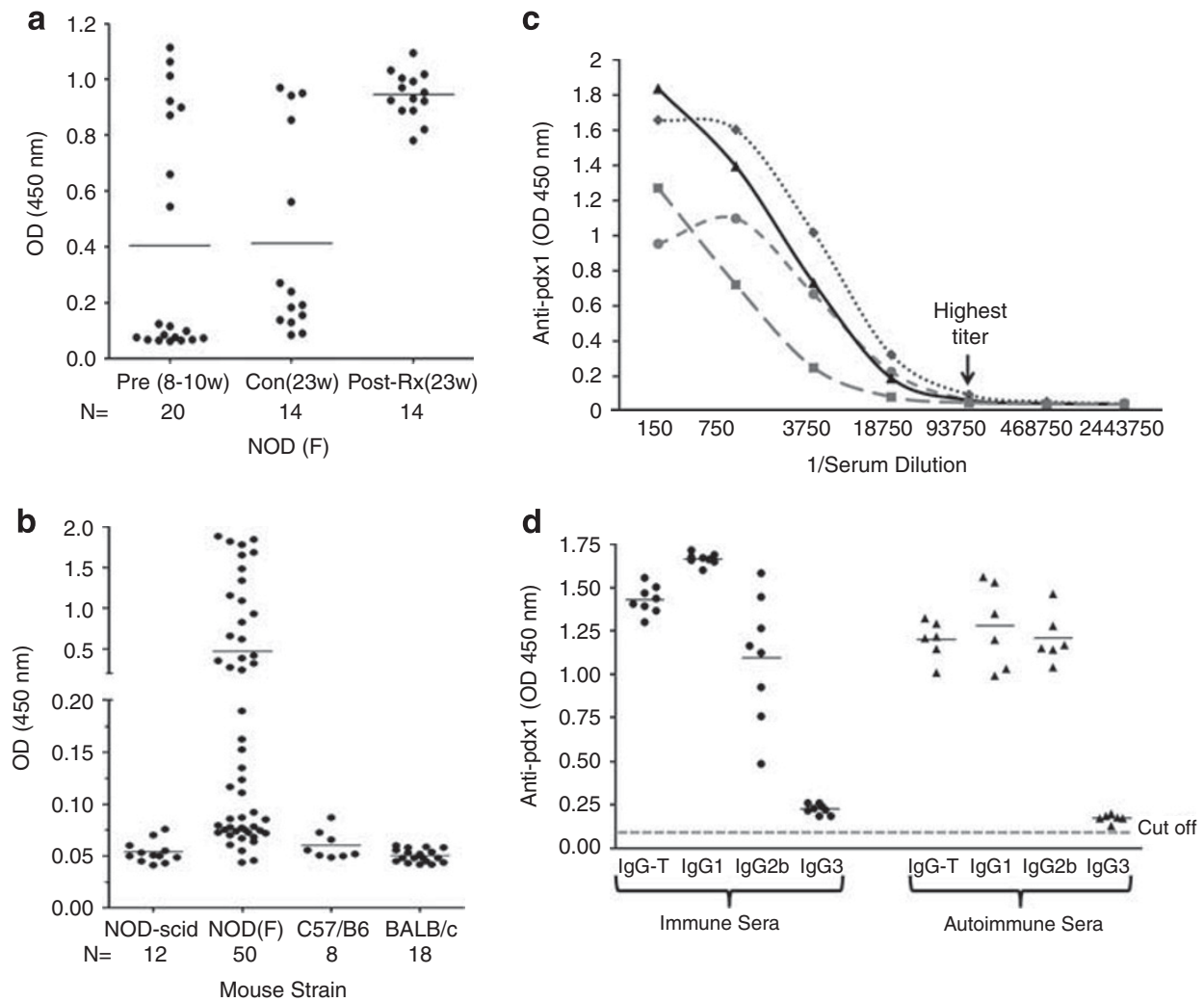

Figure $1 \mathrm{Pdx} 1$ autoantibodies in prediabetic NOD mice. (a) Detection of Pdx1 antibodies in NOD mice. Serum samples were collected from female NOD mice at various ages ranging from 8 to 23 weeks or NOD mice treated with daily injection of rPdx1 for 12 weeks. PAAs were assayed using ELISA at a serum dilution of 1:30. Positive value is defined 3 s.d. above the mean of the BALB/c control sera (Pre, pretreatment; Con, PBS-treated controls; Post-Rx, treated with rPdx1). (b) Comparison of PAAs in various strains of mice. Serum samples from various mouse strains at different ages were collected and PAAs were measured by ELISA and expressed as OD values at $450 \mathrm{nM}$ using the 99 th percentile of BALB/c control mice as the threshold definition of positivity. (c) PAAs titration curve. Selected PAA-positive serum samples at the age of 10 weeks were serially diluted as indicated and OD values were determined by ELISA. Titer is defined as the last dilution giving positive OD reading above control. (d) Isotypes of anti-Pdx1 antibodies. Total anti-Pdx1 antibody activity (IgG-T) and anti-Pdx1 antibodies of the $\lg \mathrm{G} 1$, IgG2b, and $\lg \mathrm{G} 3$ isotypes in serum samples from NOD mice immunized with Pdx1 (immune sera, solid circles) and selected PAA-positive autoimmune sera (triangle) from NOD mice were assayed by ELISA (1:30 dilution). An OD of 0.10 was used as the positive cutoff value ( $\geqslant 3$ s.d. above the mean of the BALB/c control sera).

an inverse correlation over time between PAA levels and blood glucose levels in individual mouse. However, some mice maintained high levels of the PAAs after the onset of diabetes. Figure 3 illustrates the relationship between levels of PAAs and blood glucose in four mice. In three mice, PAAs peaked before the onset of diabetes. In the fourth mouse (m19L), low levels of PAAs were seen consistently without an apparent peak; this mouse remained normoglycemic at 25 weeks. These results suggest that PAAs predict the onset of T1D in NOD mice.

\section{B-Cell Autoepitopes are Sensitive for Partial Trypsin Treatment}

As western blot analysis showed that ELISA-positive NOD mouse sera recognized primarily the full-length $\mathrm{Pdx} 1$, we examined whether these sera might recognize a common epitope. We first partially digested the full-length $\mathrm{rPdx} 1$ by incubating with trypsin. As shown in Figure 4, $10 \mathrm{~min}$ digestion of Pdx1 generated multiple fragments that were stained by Coomassie blue (left panel). Interestingly, two
NOD mouse samples (m2L and $\mathrm{m} 7 \mathrm{R}$ ) recognized the slowest migrating tryptic fragment of $\mathrm{Pdx} 1$ as well as full-length $\mathrm{Pdx} 1$.

In addition, we also screened 37 serum samples from patients $(n=25)$ with well-established T1D or anti-insulin autoantibody (IAA)-positive individuals $(n=12)$ for the presence of anti-Pdx1 antibodies by western blotting using purified full-length rat Pdx1 protein. Six serum samples were immunoreactive with Pdx1 protein: two samples (2/25) were from patients with long-standing T1D and four samples (4/12) from individuals with a positive test for IAA. To localize the immunodominant epitopes, partially trypsindigested Pdx1 protein was used as antigen. As shown in Figure 4a, human serum (H11) from a T1D patient recognized only full-length $\mathrm{Pdx} 1$ (more details below), whereas rabbit anti-Pdx1 polyclonal immune serum was immunoreactive with nearly all of the fragments. Thus, the major autoepitope(s) recognized by NOD and human T1D sera are likely to be located near the C-terminus of Pdxl, as 19 out of 29 of the trypsin-cleavage sites (arginine or lysine) are located within amino acids 160-283. 

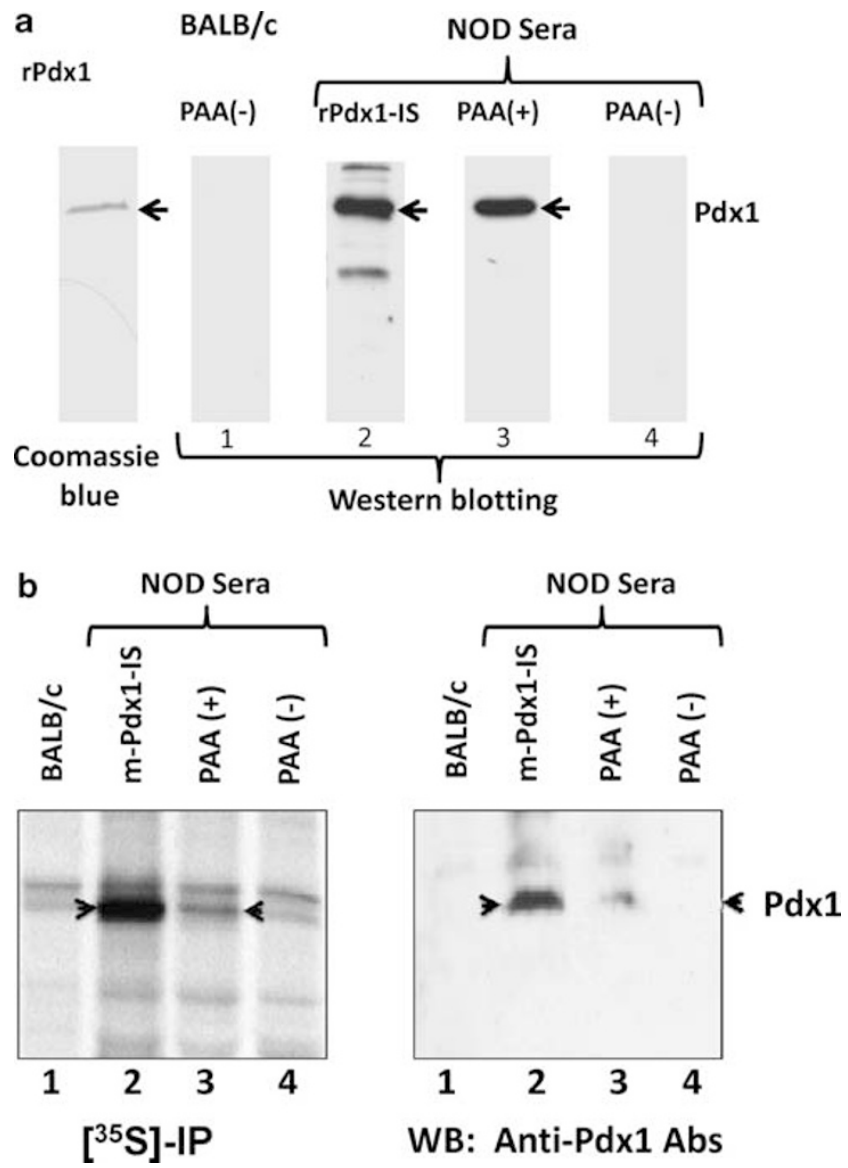

Figure 2 Confirmation of PAAs by (a) western blot and (b) immunoprecipitation. (a) Western blotting. Purified recombinant rat Pdx 1 protein ( $1 \mu \mathrm{g} /$ lane) was separated on a $10 \%$ SDS-PAGE and stained with Coomassie blue or transferred to PVDF membrane. The membrane was probed with $B A L B / c$ or NOD mouse serum either positive $(P A A+)$ or negative (PAA-) by ELISA, or with rabbit polyclonal anti-Pdx1 immune serum ( $\mathrm{rPdx} 1-\mathrm{IS})$ as a positive control. (b) Immunoprecipitation and western blotting. Rat insulinoma cells (INS-1) were labeled with $\left[{ }^{35} \mathrm{~S}\right]$ methionine overnight and cell lysate $(0.5 \mathrm{mg})$ was incubated for $1 \mathrm{~h}$ at $4{ }^{\circ} \mathrm{C}$ with preformed immune complexes by incubating $10 \mu \mathrm{l}$ mouse serum with $50 \mu \mathrm{l}$ protein A/G-Sepharose overnight at $4{ }^{\circ} \mathrm{C}$. $\left[{ }^{35} \mathrm{~S}\right]$-labeled proteins were separated by SDS-PAGE, fluorographed, and the dried gel was exposed to $\mathrm{X}$-ray film at $-80^{\circ} \mathrm{C}$ for 7 days. In parallel, unlabeled INS- 1 cell lysate was subjected to immunoprecipitation with the same mouse sera. The immune complexes were separated by SDS-PAGE, transferred to PVDF membrane, and probed with rabbit anti-Pdx1 polyclonal antibodies (1:2000). Lanes 1, $\mathrm{BALB} / \mathrm{c} ; 2$, Pdx1-treated NOD mouse immune serum (m-Pdx1-IS); 3, $\mathrm{PAA}(+)$; and 4, PAA $(-)$ NOD serum samples. Arrow indicates position of $\mathrm{Pdx} 1$ protein.

\section{B-Cell Autoepitopes are Located on the C-Terminal Portion of Pdx1}

To map the B-cell autoepitopes, we constructed, expressed, and purified histidine-tagged C-terminal truncated Pdx1 proteins consisting of amino acids $1-119,1-159$, and 1-199 (Figure $4 \mathrm{~b}$, upper panel). These truncated proteins were mixed with full-length $\mathrm{rPdx} 1$ for western blotting using three NOD-PAA + mouse serum samples (Figure $4 \mathrm{c}$, left panel) and six human PAA + serum samples (Figure 4c, right panel, described below). NOD mouse sera recognized primarily fulllength $\mathrm{rPdx} 1$ protein (a), with weaker binding (7R) to the truncated Pdx1 protein (b, 1-199), but not the two shorter truncated Pdx1 proteins $c(1-159)$ and $d(1-119)$. In contrast, the rabbit polyclonal anti-Pdx1 immune serum (rPdx1 IS) recognized all four proteins. Serum from a negative NOD mouse did not react with any of the proteins (data not shown), suggesting that the PAAs in mouse sera reacted with a major epitope (I) located within the C-terminal 84 amino acid residues and a minor epitope (II) located within Pdx1 (160-199).

To exclude reactivity with the His-Tag or the possibility that amino acids 1-199 participate in forming a discontinuous epitope, we inserted a cDNA fragment encoding the C-terminal 84 amino acids (200-283) into the pGRx5.1 GST expression system. Using this protein without cleavage from GST, we carried out western blots, showing that autoimmune NOD sera bind strongly to the GST-Pdx1/200-283 or p83 protein but not to GST (Figure 4d, upper panel), indicating that the major autoepitope indeed is located on the C-terminal portion of Pdx1 (amino acids 200-283).

\section{Identification of Epitope(s) for Human PAAs}

To determine whether PAAs from human sera recognize the same epitope bound by NOD mouse PAAs, a mixture of Pdx1 and its truncated forms was used for western blotting using PAA + human sera of T1D patients and IAA-positive individuals. Figure $4 \mathrm{c}$ (top right panel) shows two patterns among the six serum samples tested. Similar to mouse autoimmune sera, the human sera in lanes 1, 2, and 3 recognized only full-length $\mathrm{Pdx} 1$, indicating that they bind to an epitope or epitopes within the C-terminal 84 amino acids. Interestingly, human sera in lanes 4, 5, and 6 reacted equally with the top two bands, consistent with the existence of another epitope (II) located within amino acids 160-199. Figure 4c (lower panel) illustrates the two putative Pdx1 autoepitopes recognized by human sera. To confirm the locations of these epitopes, we inserted cDNA encoding Pdx1 amino acids 160-199 (as p40) into the pGRx5.1 GST expression system. Using this fusion protein, we carried out western blots, showing that human sera (Figure 4c, lanes 4, 5, and 6) bind strongly to the GST-p40 protein and full-length Pdx1, but not to GST-p83 or GST alone, indicating that autoepitope II indeed is located within amino acids 160-199 of Pdx1 (Figure 4d, lower panel).

\section{Pdx1-Stimulated In Vitro T-Cell Proliferation}

To determine whether Pdx1 can stimulate antigen-specific T-cell proliferation, freshly isolated splenocytes from newonset diabetic NOD mice were stimulated with various forms of Pdx1 protein including full-length $\mathrm{Pdx} 1$ and different truncated forms. Anti-CD3 antibodies were used as a positive control for T-cell proliferation, and as a negative control we used either untreated or GST-treated (unrelated protein) 

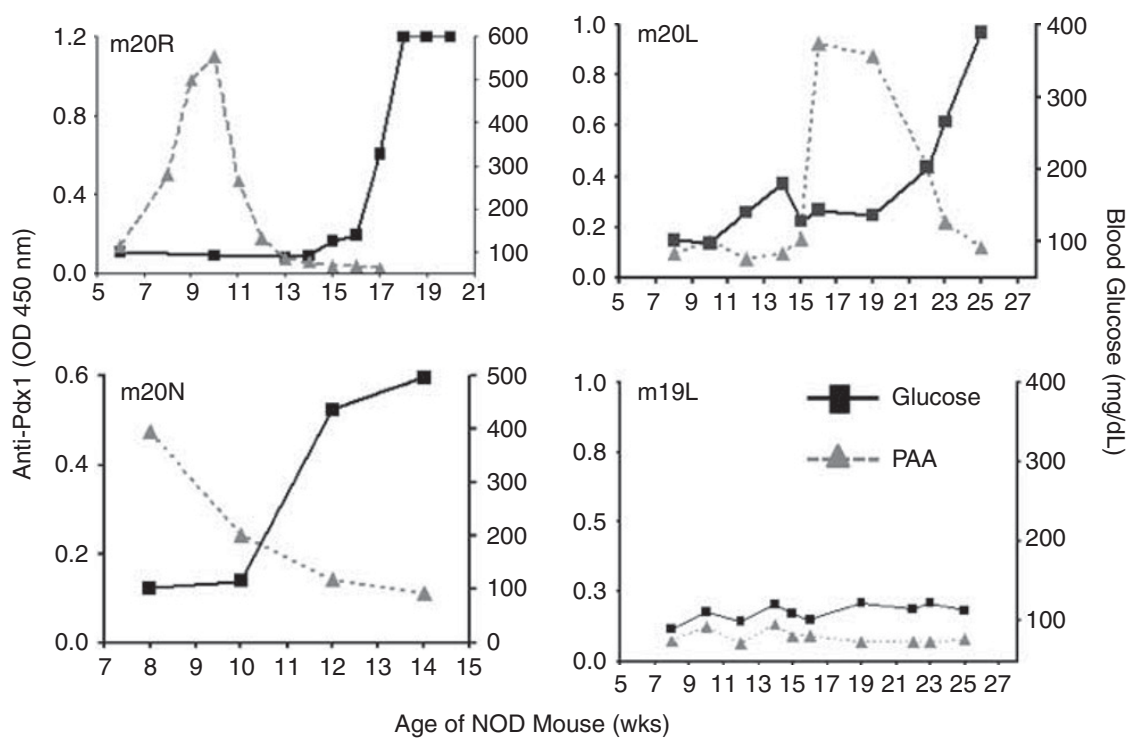

Figure 3 Relationship between PAA levels and the onset of diabetes. Serum samples were collected biweekly from 5-week-old NOD mice ( $n=20)$. PAAs were detected by ELISA and expressed as OD values at $450 \mathrm{nM}$. Blood glucose levels were monitored weekly through tail vein snipping. Four representative mice $(\mathrm{m} 20 \mathrm{R}, \mathrm{m} 20 \mathrm{~L}, \mathrm{~m} 20 \mathrm{~N}$, and $\mathrm{m} 19 \mathrm{~L})$ are shown here.

T cells. Figure 5a shows that full-length as well as truncated forms of Pdx1 can stimulate T-lymphocyte proliferation. However, the C-terminus of Pdx1 (200-283, or p83) had the most potent T-cell stimulatory effect (almost sevenfold increase over control GST, $P<0.001$ by Student's $t$-test), suggesting that the major T-cell epitope(s) are located in close proximity to the major B-cell autoepitope. Interestingly, a significant T-cell stimulatory effect also was observed in the wells containing p200 (four times over control, $P<0.001$ by Student's $t$-test), supporting the possible presence of minor autoepitopes within the region of p160-200.

\section{DISCUSSION}

Autoantibodies to islet cell antigens and autoantigen-reactive T-cells are hallmarks of T1D. ${ }^{1,18,19}$ We report here that Pdx1 is a novel pancreatic $\beta$-cell-specific autoantigen recognized by both B- and T-cells in NOD mice. Through unknown mechanisms Pdx1 protein from the pancreatic islet $\beta$-cells may stimulate the generation of the Pdx1-epitope-specific autoantibodies that can be detected by ELISA, western blotting, and IP assays. Although PAAs are found in both prediabetic and diabetic NOD mouse sera, the levels generally peak before the onset of diabetes. PAAs were only found in NOD mice, and were not present in congenic NOD-scid mice or non-autoimmune strains such as $\mathrm{C} 57 \mathrm{BL} / 6$ and $\mathrm{BALB} / \mathrm{c}$. The frequency of PAAs in 5- to 25-week-old prediabetic mice was over 50\%. Using ELISA, PAAs were detectable in 5-week-old NOD mice, indicating that these antibodies are an early disease marker. Although variable, the onset of PAA production was generally before the onset of diabetes. Interestingly, autoantibody levels declined substantially or even disappeared with the onset of diabetes. The peak titers are relatively high, up to $1 / 20000$ or more by ELISA, suggesting that PAAs may be useful for predicting the onset of diabetes in NOD mice.

Currently, IAAs are the most specific predictor of the onset of diabetes. ${ }^{20-23}$ However, these autoantibodies generally are found at very low levels. In contrast, the titers of PAAs were considerably higher than those reported previously for IAA, ${ }^{20}$ suggesting that for diabetes susceptibility screening, assays for PAAs may afford an improved signal/noise ratio over assays for IAA at least in mice. Consistent with the onset of IAA, ${ }^{20}$ we detected PAAs at five weeks of age. The prevalence of PAAs in prediabetic NOD mice was comparable with that of IAAs. ${ }^{23}$ In a collaborative international workshop study, IAAs were detected by ELISA in NOD mice at frequencies of $0 \%$ at 4 weeks, $14 \%$ at 8 weeks, $19 \%$ at 12 weeks, and $42 \%$ at the onset of diabetes. ${ }^{23}$ Using a more sensitive radiobinding assay, IAAs were detected at the onset of diabetes in 15-75\% of NOD mice. ${ }^{23}$ In this study, PAAs were detected at the onset of disease in $52 \%$ of NOD mice, a figure that may improve further if larger numbers of mice are studied and a more sensitive and optimized assay is used. It will be of interest in the future to determine whether the mice exhibiting PAAs and IAAs are the same or overlapping sets and whether measuring both specificities enhances the predictive value of autoantibody testing in NOD mice and for T1D patients.

Analysis of the fine specificities of murine PAAs showed the presence of an immunodominant epitope or epitopes on the C-terminal 83 amino acids of the Pdx1 protein. The NOD mouse sera react strongly with GST-p83 (Pdx1 amino acids 200-283), indicating that sequences outside this region are not required for epitope folding. Pdx1, a highly conserved protein of 283 amino acids, contains a transactivation 


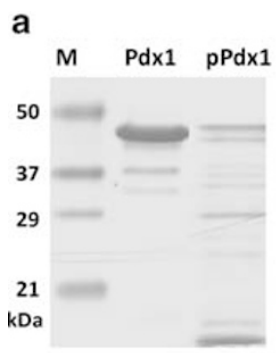

Coomassie blue

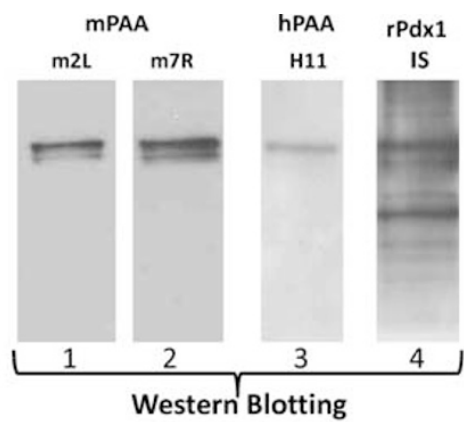

hPAA
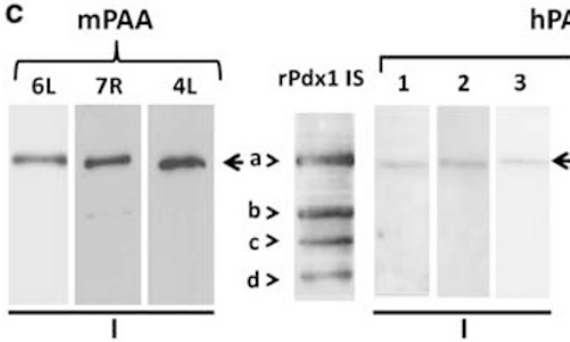

1

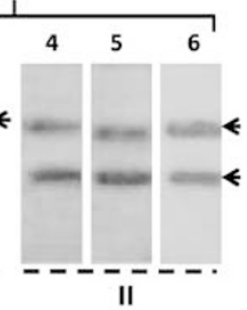

Full length and C-truncated Pdx1 proteins

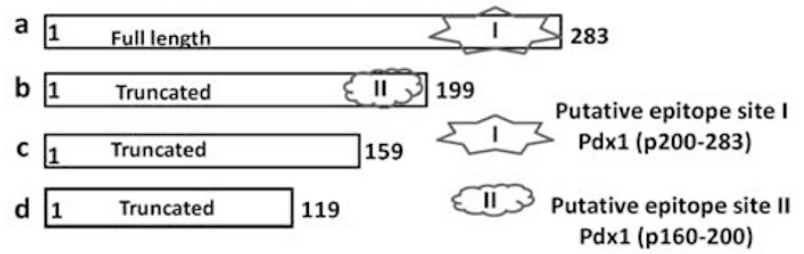

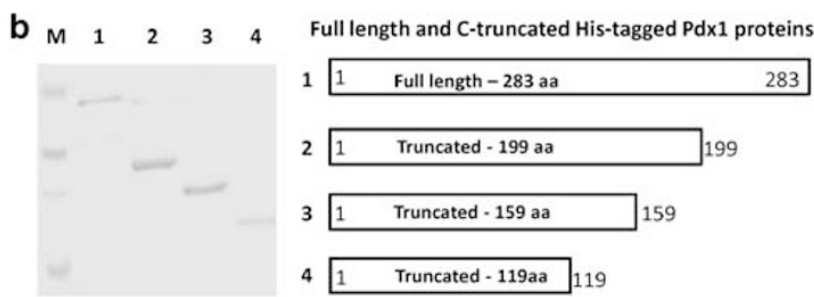
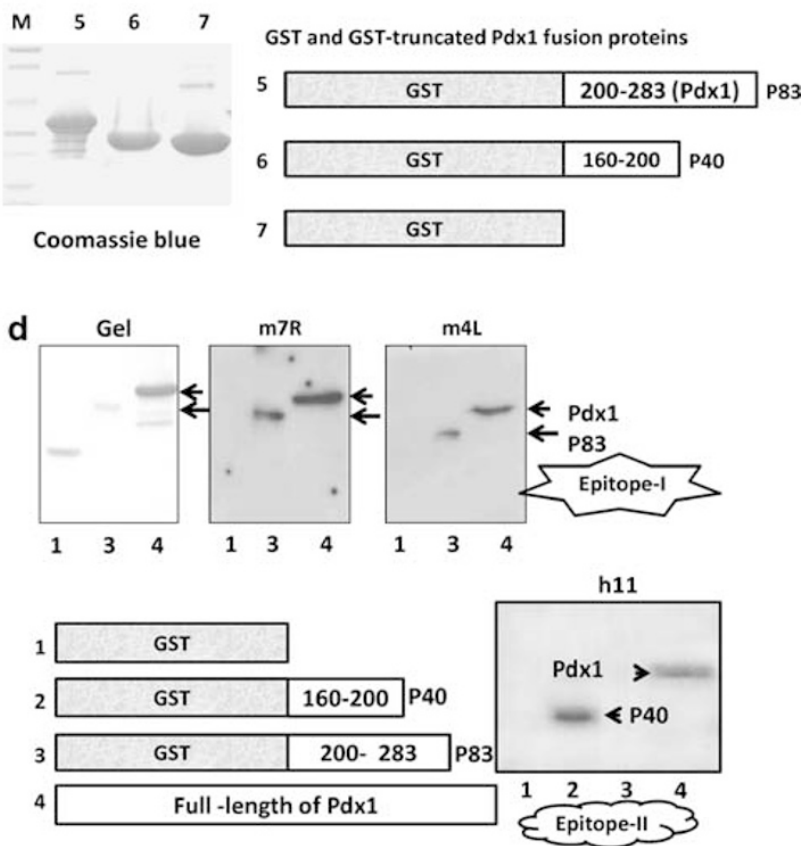

Figure 4 Identification of immunodominant B-cell autoepitopes of Pdx1. (a) Partial digestion of Pdx1 and western blotting. Pdx1 protein was partially digested with trypsin at $\mathrm{pH} 7.625^{\circ} \mathrm{C}$ for $10 \mathrm{~min}$, and the digested products were separated and stained with Coomassie blue (left panel) or transferred to the membrane for blotting with two PAA $(+)$ mouse sera $(\mathrm{m} 2 \mathrm{~L}$ and $\mathrm{m7R}$, lanes 1 and 2), human PAA + serum (H11, lane 3), or rabbit polyclonal anti-Pdx1 immune serum (lane 4). (b) Expression and purification of truncated Pdx1 proteins. Full-length or truncated Pdx 1 proteins with an $\mathrm{N}$-terminal histidine tag were prepared as described in Methods, separated by SDS-PAGE, and stained with Coomassie blue dye (upper panel). Lanes 1, full-length; 2, Pdx1 (1-199); 3, Pdx1 (1-159); and 4, Pdx1 (1-119). Lower panel shows three purified proteins GST-Pdx1 (200-283) 'p83,' GST-Pdx1 (160-200) 'p40,' and GST that were separated by SDS-PAGE and stained with Coomassie blue. Lanes 5, p83; 6, p40; and 7, GST. (c) Identification of two dominant epitope regions using a mixture of $\operatorname{Pdx} 1$ proteins. Four purified proteins, $\operatorname{Pdx} 1$ (a) and its truncated forms (b, $c$, and d), were mixed at equal concentrations and used for western blotting to detect autoantibodies. Each lane was loaded with $5 \mu \mathrm{g}$ of mixed proteins, resolved by SDS-PAGE, transferred to PVDF membrane, and probed using three mouse $\mathrm{PAA}(+)$ sera $(6 \mathrm{~L}, 7 \mathrm{R}$, and $4 \mathrm{~L})$ at a dilution of 1:1500, or six human $\mathrm{PAA}(+)$ sera (lanes $1-6)$ at a dilution of 1:200. Rabbit polyclonal anti$\mathrm{Pdx} 1$ immune serum ( $\mathrm{rPdx} 1 \mathrm{IS}$ ) was used as a positive control at a dilution of 1:2000. Arrows indicate full-length Pdx1 (a) and truncated proteins (b, c, and d). Lower panel shows the structure of Pdx1 and its truncated forms and potential autoepitope regions I and II for mouse and human PAAs. (d) Confirmation of immunodominant autoepitopes. Upper left panel shows Coomassie blue staining of GST (lane 1), p83 (lane 2), and Pdx1 (lane 3). Right two panels are immunoblots using the same amount of proteins and blotted with two NOD mouse PAA $(+)$ sera. Arrows indicate the positions of Pdx1 and p83. Lower left panel is a diagram of the four proteins (1, GST; 2, GST-p40; 3, GST-p83; and 4, Pdx1) used in this study. The lower right panel depicts an immunoblot using human $\mathrm{h} 11 \mathrm{PAA}(+)$ serum. Arrows indicate positions of corresponding proteins as indicated.

domain at the N-terminus (amino acids 1-79), a homeodomain in the central region (amino acids 146-206), and an evolutionarily conserved, C-terminal domain of unknown function. ${ }^{24}$ The latter region, which contains the major autoepitope(s) recognized by NOD mouse sera, notably contains 19 trypsin-sensitive (arginine or lysine) sites between amino acids 160 and 283, and cleavage at these sites abolishes immunoreactivity of the C-terminal domain (Figure 4a). Ten of these sites are located on amino acids 160-199 within the homeodomain of Pdx1 containing an antennapedia-like protein transduction domain, DNA-binding domain (amino acids 191-196), and nuclear localization signal (amino acids 197-203). ${ }^{16,25}$ However, this region is dispensable for immunogenicity, suggesting that some or all of the remaining nine trypsin-sensitive arginine or lysine residues located on amino acids 200-280 are important in forming the immunodominant epitope.

In contrast to NOD mice, T1D patients' sera recognize two epitopes, one of which (Epitope I) appears similar if not identical to the immunodominant epitope in NOD mice. The second autoepitope, located within the homeodomain of Pdx1 (p160-199), is recognized by a subset of human auto- 


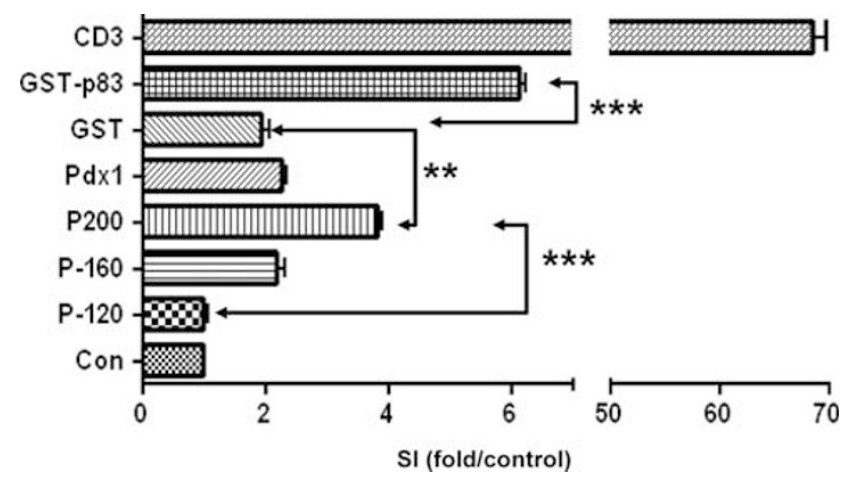

Figure 5 Antigen-specific T-cell proliferation to $\mathrm{Pdx} 1$ and its truncated forms. Splenocytes $\left(10^{6}\right.$ cells/well) isolated from a NOD mouse with newonset diabetes were incubated in a 96-well plate. $\mathrm{Pdx} 1$ and various truncated proteins were added into the wells at a final concentration of $1 \mu \mathrm{g} / \mathrm{ml}$ for $48 \mathrm{~h}$, following by adding $\left[{ }^{3} \mathrm{H}\right]$ thymidine for an additional $24 \mathrm{~h}$ Cells were captured on filter paper, washed five times, and $\left[{ }^{3} \mathrm{H}\right]$ thymidine incorporation was determined by scintillation counting. Background count is without $\left[{ }^{3} \mathrm{H}\right]$ thymidine. ${ }^{* * P}<0.01$ and ${ }^{* * *} P<0.001$ (Student's $t$-test). $\mathrm{Sl}$, stimulation index.

immune sera (Figure 4c) but also weakly by some murine sera (see Figure 4c, mouse \#7R). This region contains 10 lysine/arginine residues, but the importance of these charged sites for antigenicity remains to be determined. Trypsinsensitive lysine/arginine residues are also critical for the antigenicity of epitopes recognized by anti-histone antibodies, ${ }^{26}$ and basic amino acids are believed to be important for autoantibody recognition of the U1-70K component of U1 small nuclear ribonucleoproteins. ${ }^{27}$ In this regard, it is interesting to note that NOD mice also produce autoantibodies against U1 small nuclear ribonucleoproteins. ${ }^{28}$ The relationship, if any, between these autoantibodies and anti-PAAs remains to be determined. However, it has been proposed that long runs of charged amino acids may render certain self-antigens immunogenic. ${ }^{29}$ In addition, autoantibodies frequently recognize epitopes located on the $\mathrm{N}$ - and C-termini of proteins, ${ }^{30}$ as was the case here.

In addition to the strong humoral autoimmune response, we found that Pdx1 also is a target of cellular immunity. In T1D and other disorders, autoreactive B-cell and T-cell epitopes frequently overlap ${ }^{21,31}$ Indeed, full-length $\mathrm{Pdx} 1$, its C-terminal 83 amino acids (p83), as well as homeodomaincontaining truncated $\mathrm{Pdx} 1$ protein (p200) stimulated proliferation of splenic $\mathrm{T}$ cells from NOD mice (Figure 5), suggesting that $\mathrm{T}$ - and $\mathrm{B}$-cell epitopes may lie in close proximity within this region. Interestingly, the proliferative response to full-length $\mathrm{Pdx} 1$ was less than that to the $\mathrm{p} 83$ or p200 fragments (Figure 5). This might be due to differences in the relative efficiency of antigen processing/presentation by antigen-presenting cells, for example, because of differences in protein folding and protease cleavage in endosomes. Further mapping of the locations of the T- and B-cell epitopes is in progress.
We previously showed that treatment of Stz-induced diabetic mice with full-length $\mathrm{rPdx} 1$ reverses diabetes by stimulating pancreatic islet $\beta$-cell regeneration and reprogramming liver cells into insulin-producing cells. ${ }^{11}$ Our recent studies indicate that daily treatment of prediabetic female NOD mice with $\mathrm{rPdx} 1$ prevents the onset of diabetes in $85 \%$ of mice at 40 weeks, whereas $95 \%$ of controls became diabetic by 25 weeks (manuscript in preparation). Interestingly, when an inactive form of Pdx1 lacking the protein translocation domain ${ }^{11,16,25}$ was administered to NOD mice, it also protected against the development of diabetes (manuscript in preparation). Western blotting verified that the mutant $\mathrm{Pdx} 1 \Delta \mathrm{PTD}$ protein retained immunoreactivity with PAAs (data not shown) and protein (p83) lacking the PTD also was capable of stimulating T-cell proliferation in vitro. These findings from in vivo animal studies are consistent with the notion of Pdxl being an autoantigen that may be related to the pathogenesis of T1D in NOD mice. Consistent with that notion, the onset of diabetes in NODscid mice was delayed after passive transfer of splenocytes from Pdx1-treated NOD mice in comparison with splenocytes from PBS-treated controls (unpublished data), suggesting that $\mathrm{rPdx} 1$ may exert an immunomodulatory effect by suppressing autoreactive diabetogenic $\mathrm{T}$ cells, as previously shown by Ferber's group. ${ }^{12}$ The immunological mechanism for the phenomenon of Pdx1 treatment preventing diabetes is now under active investigation.

The presence of PAAs in sera from patients with T1D suggests that our observations in the NOD mouse model have relevance to human T1D. Among the six PAA + T1D or IAA-positive patient serum samples identified in preliminary screening studies, we discovered two distinct patterns by western blotting, suggesting the autoepitopes to human PAAs can be located either exclusively within the C-terminus or in both the C-terminus and the homeodomain of Pdx1. It will be of interest to examine both the role of immune responses to Pdx1 in the pathogenesis of human T1D and the clinical value of testing for these autoantibodies in children at risk for developing autoimmune diabetes.

T1D is caused by autoimmune islet $\beta$-cell destruction and islet $\beta$-cell antigens are the targets of this autoimmune disorder. Self-antigens specific to islet $\beta$-cells stimulate the production of autoantibodies and the generation of antigenspecific autoreactive $\mathrm{T}$ cells. These autoantigens include insulin, glutamic acid decarboxylase (GAD65), insulinomaassociated-2 antigen (IA-2), Slc30A8, ${ }^{32} \mathrm{Pdx} 1$, and others ${ }^{8} .{ }^{33}$ Although immunotherapy can prevent T1D in the NOD mouse model, we have yet to find a safe and effective way to prevent or ameliorate T1D in humans. ${ }^{34,35}$ A recent randomized trial of GAD vaccination in new-onset of T1D patients shows very limited improvement in preserving the pancreatic $\beta$-cell function, ${ }^{10}$ raising the possibility that it may be necessary to tolerize against multiple autoantigens in order to suppress immune attack. ${ }^{34,36}$ In addition to these previous reports regarding other $\beta$-cell-specific autoantigens, the 
present data suggest that combined antigen-specific immunotherapy and $\beta$-cell regeneration therapy may be a feasible therapeutic approach in T1D. ${ }^{21,37}$ From our current knowledge of Pdxl and its critical roles in pancreatic development and maintenance of $\beta$-cell function, ${ }^{38,39}$ the promotion of $\beta$-cell regeneration, ${ }^{11}$ prevention of $\beta$-cell apoptosis, ${ }^{40}$ and reprogramming of non-pancreatic cells to become insulin-producing cells, ${ }^{41-43} \mathrm{Pdx} 1$ might be a useful therapeutic agent for treating T1D.

\section{ACKNOWLEDGEMENT}

This work was supported by grants from the National Institutes of Health, NIAMS AR44731 (W Reeves), and NIDDK DK071831 and DK64054 (LJ Yang).

\section{DISCLOSURE/CONFLICT OF INTEREST}

The authors declare no conflict of interest.

1. Atkinson MA, Eisenbarth GS. Type 1 diabetes: new perspectives on disease pathogenesis and treatment. Lancet 2001;358:221-229.

2. Wasserfall $\mathrm{CH}$, Atkinson MA. Autoantibody markers for the diagnosis and prediction of type 1 diabetes. Autoimmun Rev 2006;5:424-428.

3. Miao D, Yu L, Eisenbarth GS. Role of autoantibodies in type 1 diabetes. Front Biosci 2007;12:1889-1898.

4. Pihoker C, Gilliam LK, Hampe CS, et al. Autoantibodies in diabetes. Diabetes 2005;54(Suppl 2):S52-S61.

5. Skyler JS. Prediction and prevention of type 1 diabetes: progress, problems, and prospects. Clin Pharmacol Ther 2007;81:768-771.

6. Giarratana N, Penna G, Adorini L. Animal models of spontaneous autoimmune disease: type 1 diabetes in the nonobese diabetic mouse. Methods Mol Biol 2007;380:285-311.

7. Liu $\mathrm{E}, \mathrm{Yu} \mathrm{L}$, Moriyama $\mathrm{H}$, et al. Animal models of insulin-dependent diabetes. Methods Mol Med 2004;102:195-212.

8. Aly T, Devendra D, Eisenbarth GS. Immunotherapeutic approaches to prevent, ameliorate, and cure type 1 diabetes. Am J Ther 2005;12:481-490.

9. Cernea S, Pozzilli P. New potential treatments for protection of pancreatic B-cell function in type 1 diabetes. Diabet Med 2008;25:1259-1267.

10. Ludvigsson J, Faresjo $M$, Hjorth $M$, et al. GAD treatment and insulin secretion in recent-onset type 1 diabetes. $\mathrm{N}$ Engl J Med 2008;359:1909-1920.

11. Koya V, Lu S, Sun YP, et al. Reversal of streptozotocin-induced diabetes in mice by cellular transduction with recombinant pancreatic transcription factor pancreatic duodenal homeobox-1: a novel protein transduction domain-based therapy. Diabetes 2008;57:757-769.

12. Shternhall-Ron K, Quintana FJ, Perl S, et al. Ectopic PDX-1 expression in liver ameliorates type 1 diabetes. J Autoimmun 2007;28:134-142.

13. Cao LZ, Tang DQ, Horb ME, et al. High glucose is necessary for complete maturation of pdx-1-vp16-expressing hepatic cells into functional insulin-producing cells. Diabetes 2004;53:3168-3178.

14. Satoh $M$, Reeves WH. Induction of lupus-associated autoantibodies in $\mathrm{BALB} / \mathrm{c}$ mice by intraperitoneal injection of pristane. J Exp Med 1994;180:2341-2346.

15. Satoh $M$, Reeves $W H$. Induction of lupus-associated autoantibodies in BALB/C mice by intraperitoneal injection of pristane. J Exp Med 1994;180:2341-2346.

16. Noguchi $\mathrm{H}$, Kaneto $\mathrm{H}$, Weir GC, et al. PDX-1 protein containing its own antennapedia-like protein transduction domain can transduce pancreatic duct and islet cells. Diabetes 2003;52:1732-1737.

17. Martin RM, Brady JL, Lew AM. The need for IgG2C specific antiserum when isotyping antibodies from C57BL/6 and NOD mice. J Immunol Methods 1998;212:187-192.

18. Gillespie KM. Type 1 diabetes: pathogenesis and prevention. CMAJ 2006;175:165-170.
19. Jasinski JM, Eisenbarth GS. Insulin as a primary autoantigen for type $1 \mathrm{~A}$ diabetes. Clin Dev Immunol 2005;12:181-186.

20. Michel C, Boitard C, Bach JF. Insulin autoantibodies in non-obese diabetic (NOD) mice. Clin Exp Immunol 1989;75:457-460.

21. Zhang L, Nakayama M, Eisenbarth GS. Insulin as an autoantigen in NOD/human diabetes. Curr Opin Immunol 2008;20:111-118.

22. Yu L, Robles DT, Abiru N, et al. Early expression of antiinsulin autoantibodies of humans and the NOD mouse: evidence for early determination of subsequent diabetes. Proc Natl Acad Sci USA 2000;97:1701-1706

23. Bonifacio $E$, Atkinson $M$, Eisenbarth $G$, et al. International workshop on lessons from animal models for human type 1 diabetes: identification of insulin but not glutamic acid decarboxylase or IA-2 as specific autoantigens of humoral autoimmunity in nonobese diabetic mice. Diabetes 2001;50:2451-2458.

24. Al Quobaili F, Montenarh M. Pancreatic duodenal homeobox factor-1 and diabetes mellitus type 2 (review). Int J Mol Med 2008:21:399-404.

25. Noguchi $H$, Matsushita $M$, Matsumoto $S$, et al. Mechanism of PDX-1 protein transduction. Biochem Biophys Res Commun 2005:332:68-74.

26. Thomas JO, Wilson CM, Hardin JA. The major core histone antigenic determinants in systemic lupus erythematosus are in the trypsinsensitive regions. FEBS Lett 1984;169:90-96.

27. James JA, Scofield RH, Harley JB. Basic amino acids predominate in the sequential autoantigenic determinants of the small nuclear $70 \mathrm{~K}$ ribonucleoprotein. Scand J Immunol 1994;39:557-566.

28. Baxter AG, Horsfall AC, Healey D, et al. Mycobacteria precipitate an SLE-like syndrome in diabetes-prone NOD mice. Immunology 1994:83:227-231.

29. Brendel V, Dohlman J, Blaisdell BE, et al. Very long charge runs in systemic lupus erythematosus-associated autoantigens. Proc Natl Acad Sci USA 1991;88:1536-1540.

30. Pollard KM, Cohen MG. Predicting antigenic determinants of autoantigens. Autoimmunity 1990;5:265-275.

31. Di Lorenzo TP, Peakman M, Roep BO. Translational mini-review series on type 1 diabetes: systematic analysis of $T$ cell epitopes in autoimmune diabetes. Clin Exp Immunol 2007;148:1-16.

32. Wenzlau JM, Moua O, Sarkar SA, et al. SIC30A8 is a major target of humoral autoimmunity in type 1 diabetes and a predictive marker in prediabetes. Ann N Y Acad Sci 2008;1150:256-259.

33. Devendra D, Yu L, Eisenbarth GS. Endocrine autoantibodies. Clin Lab Med 2004:24:275-303.

34. Cernea S, Herold KC. Drug insight: new immunomodulatory therapies in type 1 diabetes. Nat Clin Pract Endocrinol Metab 2006;2:89-98.

35. Harrison LC. The prospect of vaccination to prevent type 1 diabetes. Hum Vaccin 2005:1:143-150.

36. Faustman DL. Immunotherapy on trial for new-onset type 1 diabetes. N Engl J Med 2008:359:1956-1958.

37. Mukherjee R, Wagar D, Stephens TA, et al. Identification of CD4+ T cell-specific epitopes of islet-specific glucose-6-phosphatase catalytic subunit-related protein: a novel beta cell autoantigen in type 1 diabetes. J Immunol 2005;174:5306-5315.

38. Offield MF, Jetton TL, Labosky PA, et al. PDX-1 is required for pancreatic outgrowth and differentiation of the rostral duodenum. Development 1996;122:983-995.

39. Kushner JA, Ye J, Schubert $M$, et al. Pdx1 restores beta cell function in Irs2 knockout mice. J Clin Invest 2002;109:1193-1201.

40. Johnson JD, Ahmed NT, Luciani DS, et al. Increased islet apoptosis in Pdx1+/- mice. J Clin Invest 2003;111:1147-1160.

41. Ferber $\mathrm{S}$, Halkin $\mathrm{A}$, Cohen $\mathrm{H}$, et al. Pancreatic and duodenal homeobox gene 1 induces expression of insulin genes in liver and ameliorates streptozotocin-induced hyperglycemia. Nat Med 2000;6:568-572.

42. Tang DQ, Cao LZ, Chou W, et al. Role of Pax4 in Pdx1-VP16-mediated liver-to-endocrine pancreas transdifferentiation. Lab Invest 2006;86:829-841.

43. Kojima $\mathrm{H}$, Fujimiya $M$, Matsumura $\mathrm{K}$, et al. NeuroD-betacellulin gene therapy induces islet neogenesis in the liver and reverses diabetes in mice. Nat Med 2003;9:596-603. 